\title{
Measurement of Residual Dipolar Couplings using Magnetically Aligned and Flipped Nanodiscs
}

\section{Thirupathi Ravula ${ }^{a, b}$ and Ayyalusamy Ramamoorthy ${ }^{a^{*}}$}

${ }^{a}$ Biophysics and Department of Chemistry, Biomedical Engineering, Macromolecular Science and Engineering, The University of Michigan, Ann Arbor, MI 48109-1055, USA.

${ }^{b}$ National Magnetic Resonance Facility at Madison, Department of Biochemistry, University of Wisconsin, Madison, WI 53706-1544, USA. 


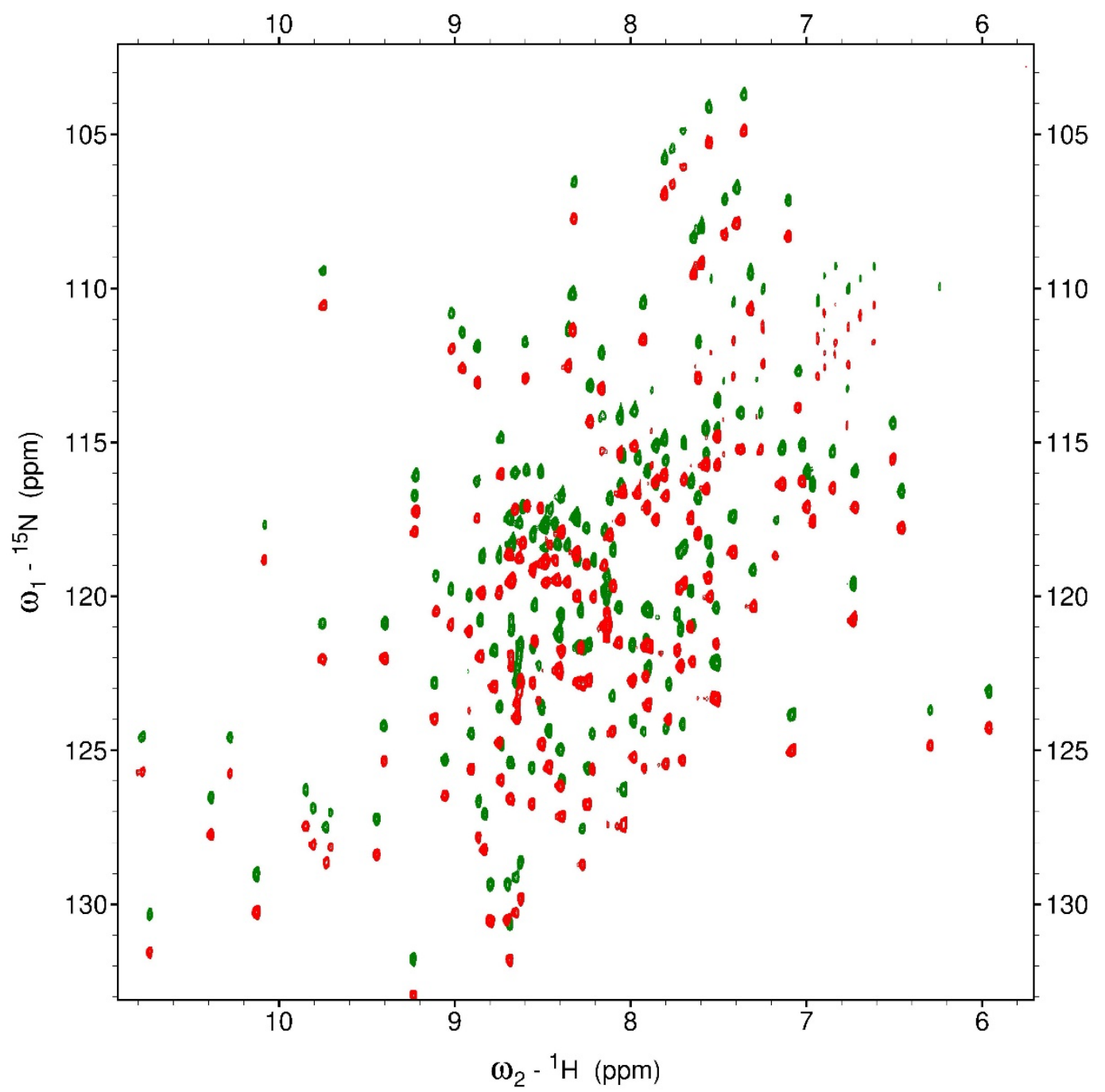

Figure S1. NMR spectra of ${ }^{15} \mathrm{~N}$-trFBD in solution. 2D IPAP-HSQC NMR spectra of $150 \mu \mathrm{M}^{15} \mathrm{~N}-$ trFBD in $10 \% \mathrm{D}_{2} \mathrm{O}, 10 \mathrm{mM} \mathrm{Kpi}, 100 \mathrm{mM} \mathrm{NaCl}, \mathrm{pH} 7.4$ and at $35^{\circ} \mathrm{C}$. 


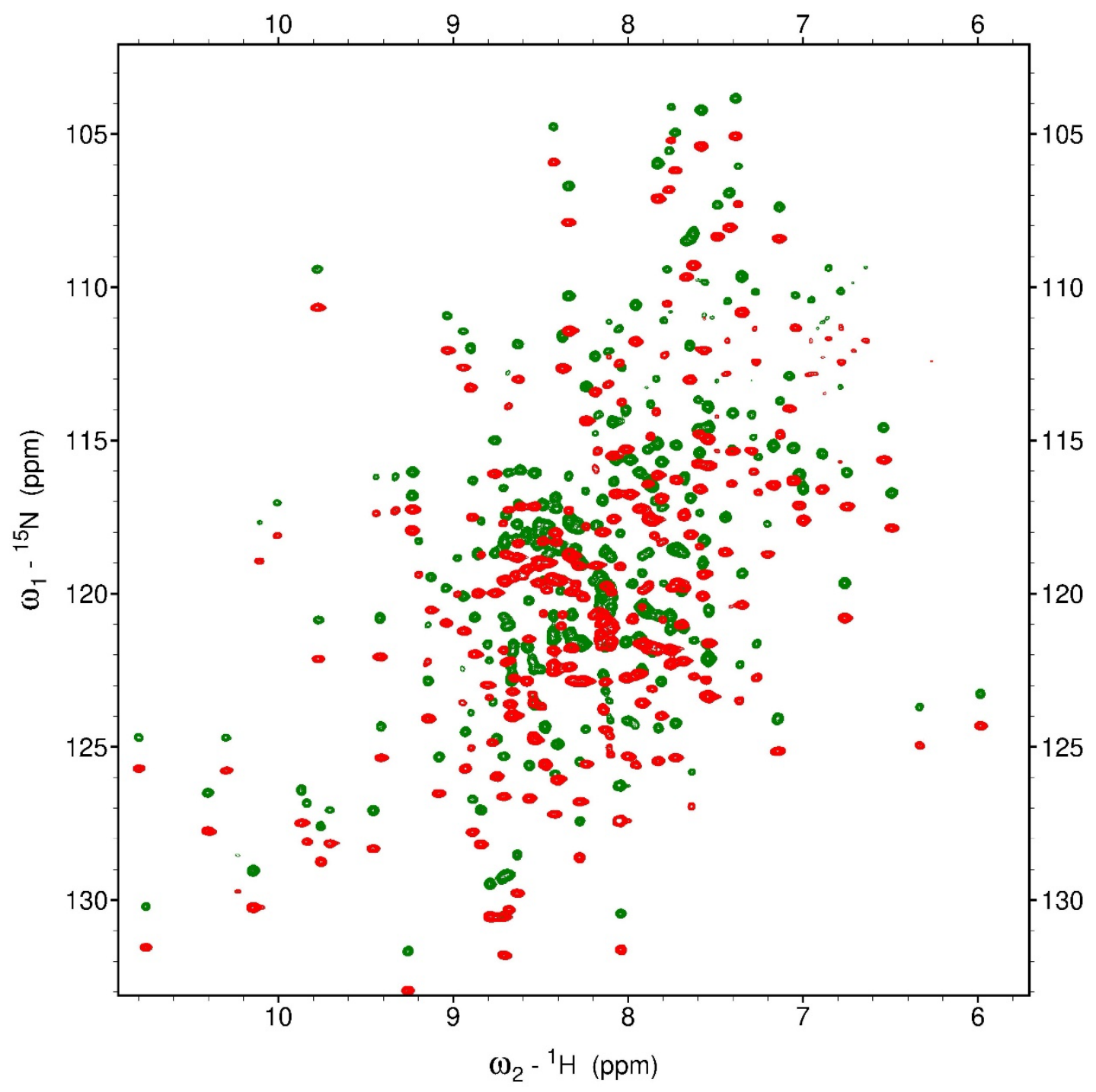

Figure S2. NMR spectra of ${ }^{15} \mathrm{~N}$-trFBD in magnetically-aligned polymer nanodiscs. 2D IPAPHSQC NMR spectra of $150 \mu \mathrm{M}^{15} \mathrm{~N}$-trFBD in the presence of 1:1 w/w DMPC-DMPE-DTPA:SMAEA nanodiscs with $40 \mathrm{mg} / \mathrm{mL}$ lipid concentration, $10 \% \mathrm{D}_{2} \mathrm{O}, 10 \mathrm{mM} \mathrm{Kpi,} 100 \mathrm{mM} \mathrm{NaCl}, \mathrm{pH} 7.4$, and at $35^{\circ} \mathrm{C}$. A molar ratio of 95:5 DMPC:DMPE-DTPA was used. The nanodiscs were magneticallyaligned with the lipid bilayer-normal oriented perpendicular to the external magnetic field direction. 


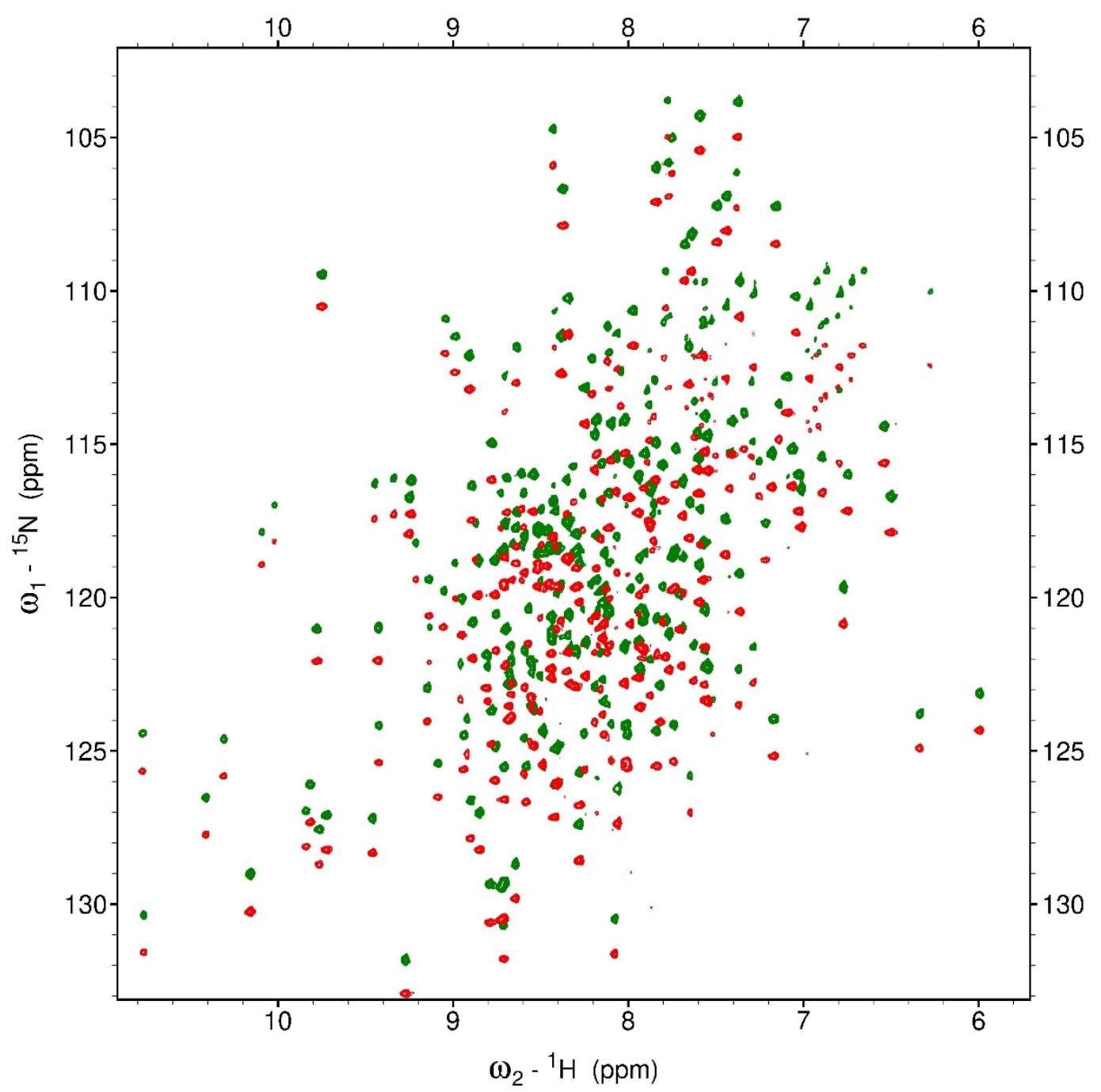

Figure S3. NMR spectra of ${ }^{15} \mathrm{~N}$-trFBD in magnetically-aligned \& flipped polymer nanodiscs. 2D IPAP-HSQC NMR spectra of $150 \mu \mathrm{M}{ }^{15} \mathrm{~N}$-trFBD in the presence of 1:1 w/w DMPC-DMPEDTPA-Yb ${ }^{3+}$ :SMAEA nanodiscs with $40 \mathrm{mg} / \mathrm{mL}$ lipid concentration, $10 \% \mathrm{D}_{2} \mathrm{O}, 10 \mathrm{mM} \mathrm{Kpi}, 100 \mathrm{mM}$ $\mathrm{NaCl}, \mathrm{pH} 7.4$, and at $35^{\circ} \mathrm{C}$. A molar ratio of 95:5 DMPC:DMPE-DTPA was used. The nanodiscs were magnetically-aligned with the lipid bilayer-normal oriented parallel to the external magnetic field direction. 

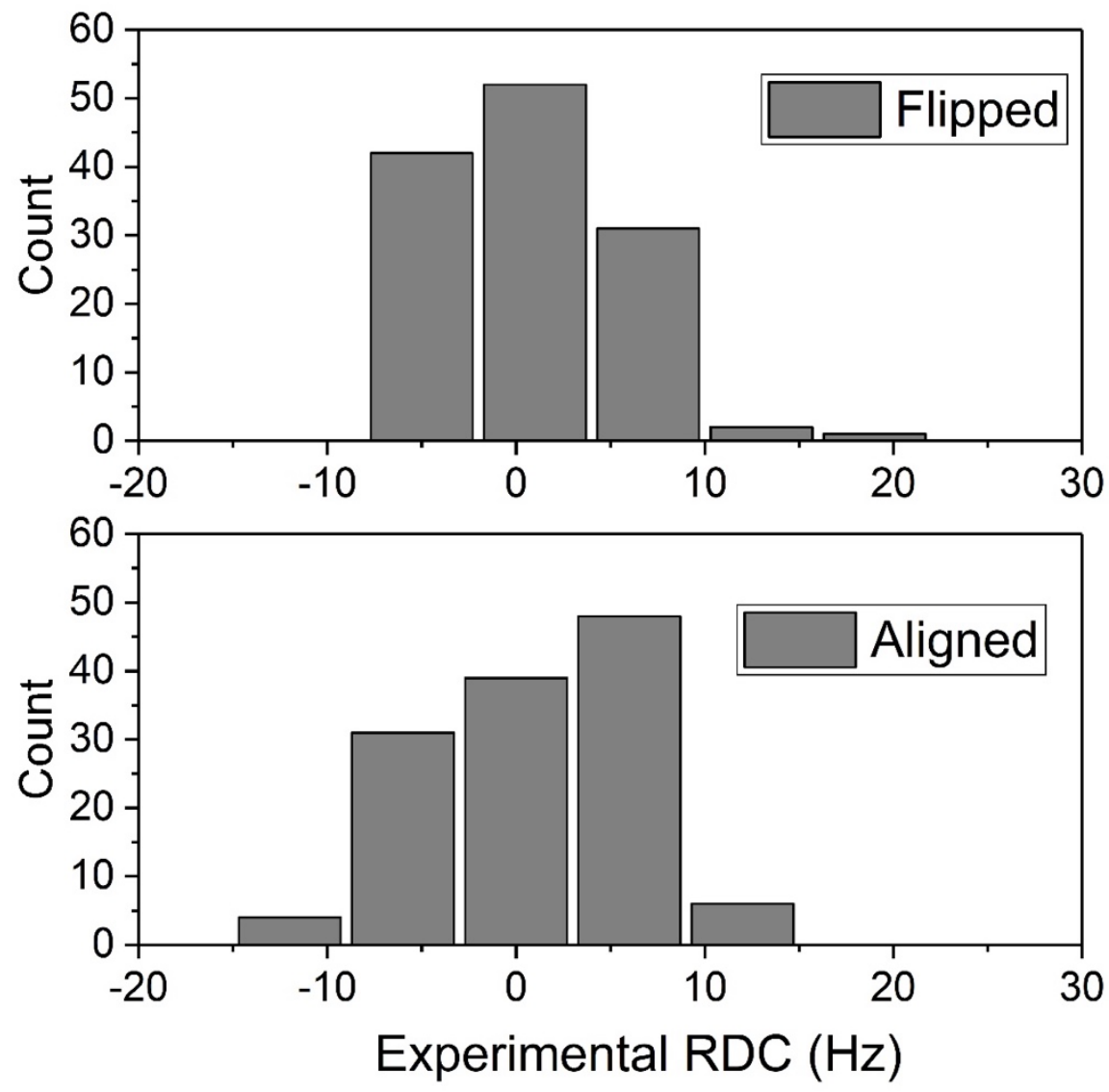

Figure S4. Comparison of RDCs measured from ${ }^{15} \mathrm{~N}$-trFBD in aligned and flipped nanodiscs. Histogram plots showing the distribution RDCs measured from NMR spectra (Figures S2 and S3) of ${ }^{15} \mathrm{~N}$-trFBD in aligned (Figure S2) and flipped (Figure S3) nanodiscs. 

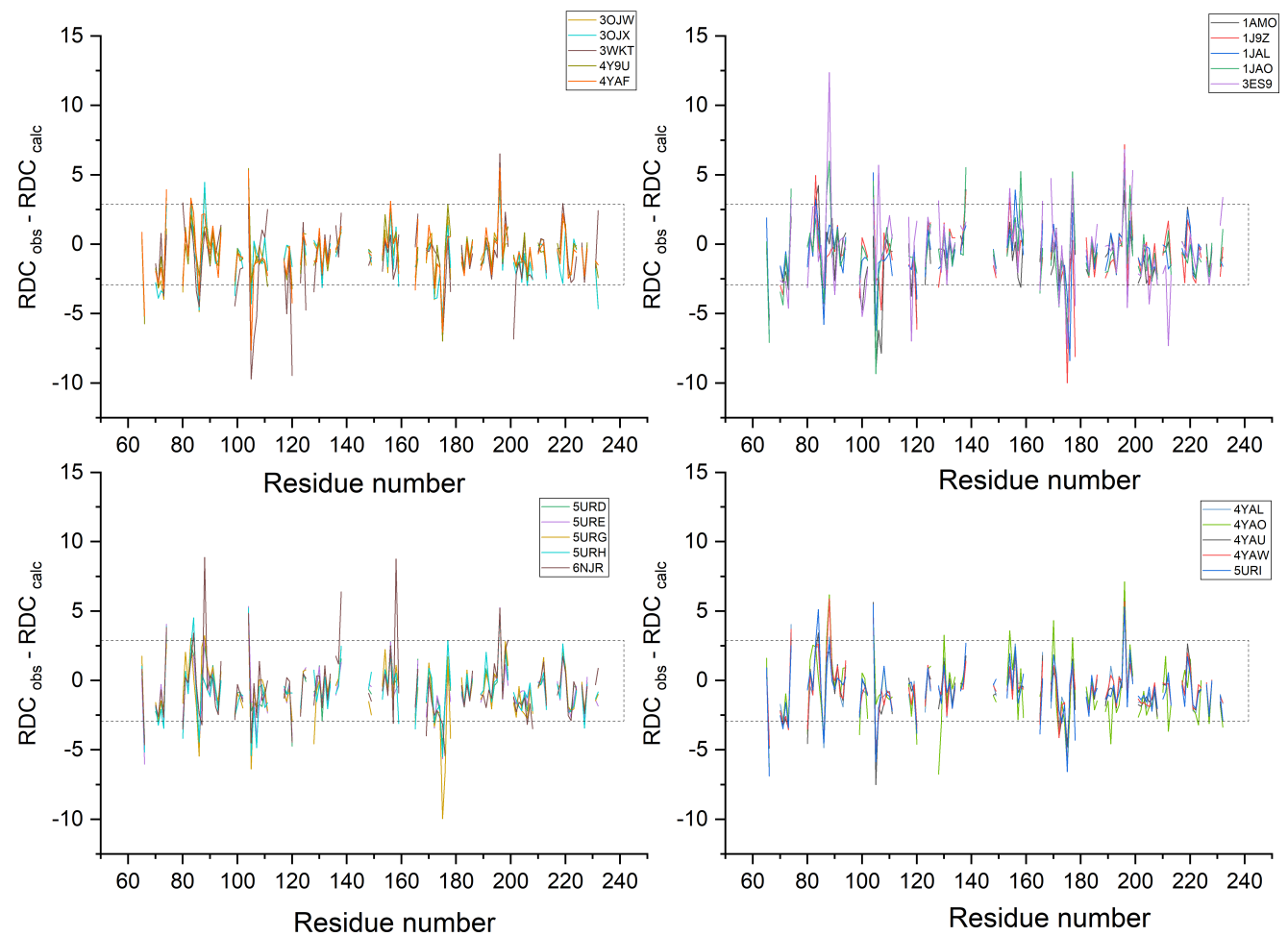

Figure S5: Plots showing the deviation of the difference in the RDC values for truncated-FBD. These values are the difference between the experimentally measured RDCS (using DMPCSMA-EA nanodiscs) and the calculated RDCs using various crystal structures mentioned in Table $\mathrm{S} 1$. These values are also given in Table S2. Most of the deviations are within a range of $\pm 3 \mathrm{~Hz}$ as indicated with dashed horizontal lines. 


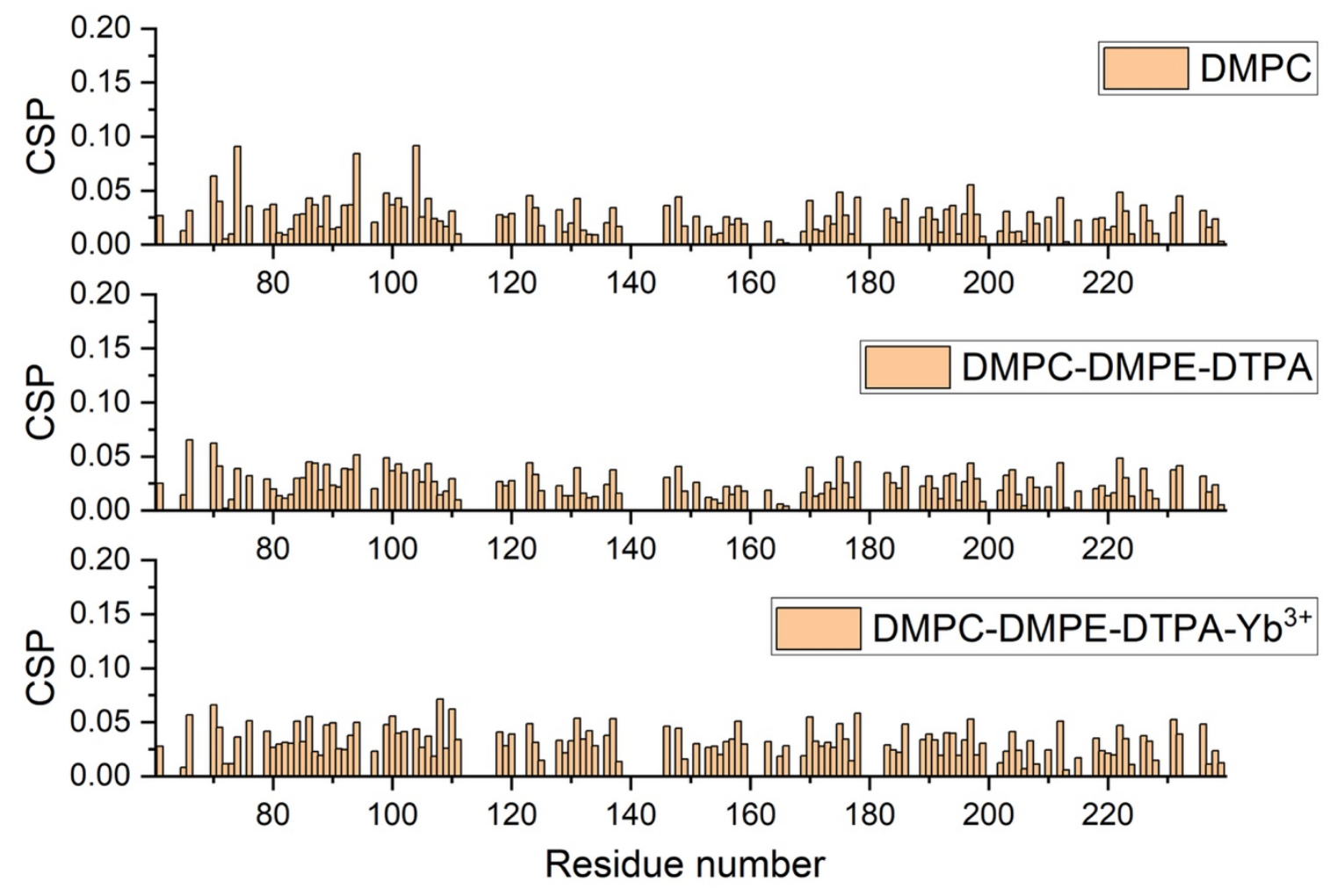

Figure S6: Chemical shift perturbation (CSPs) measured from ${ }^{1} \mathrm{H}-{ }^{15} \mathrm{~N}$ HSQC NMR spectra of ${ }^{15} \mathrm{~N}$-labeled truncated-FBD in the absence (i.e. FBD in buffer with no nanodiscs) and presence of DMPC-SMA-EA, DMPC-DMPE-DTPA-SMA-EA and DMPC-DMPE-DTPA-Yb3+-SMA-EA nanodiscs. The CSP are calculated from the equation CSP $=\sqrt{(\Delta 1 H)^{2}+0.128(\Delta 15 N)^{2}}$. 


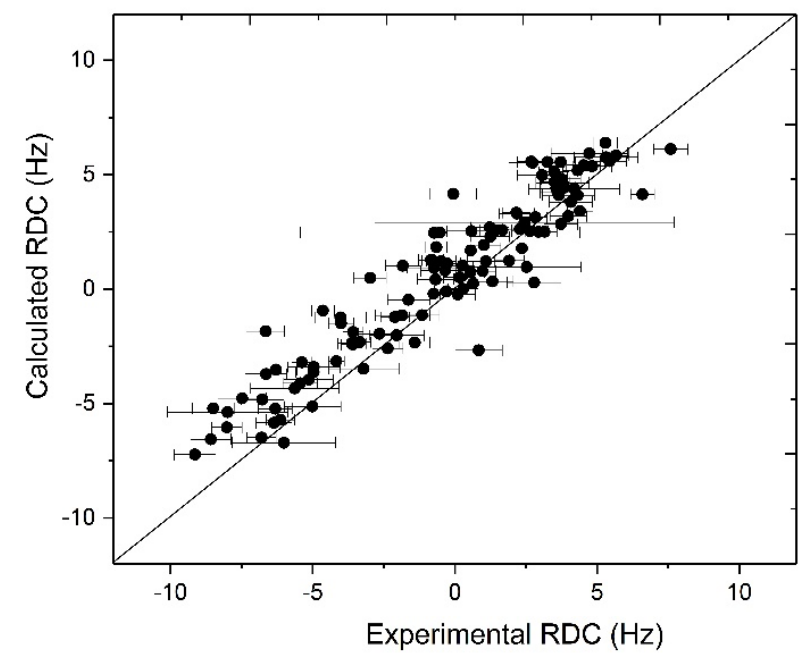

Figure S7. Correlation of experimentally measured and calculated RDCs for ${ }^{15} \mathrm{~N}$-trFBD in DMPCDMPE-DTPA-Yb ${ }^{3+}$-SMA-EA nanodiscs. The calculated RDCs were obtained using the PDB structure $4 \mathrm{Y} 9 \mathrm{U}$ and PALES software. ${ }^{1}$
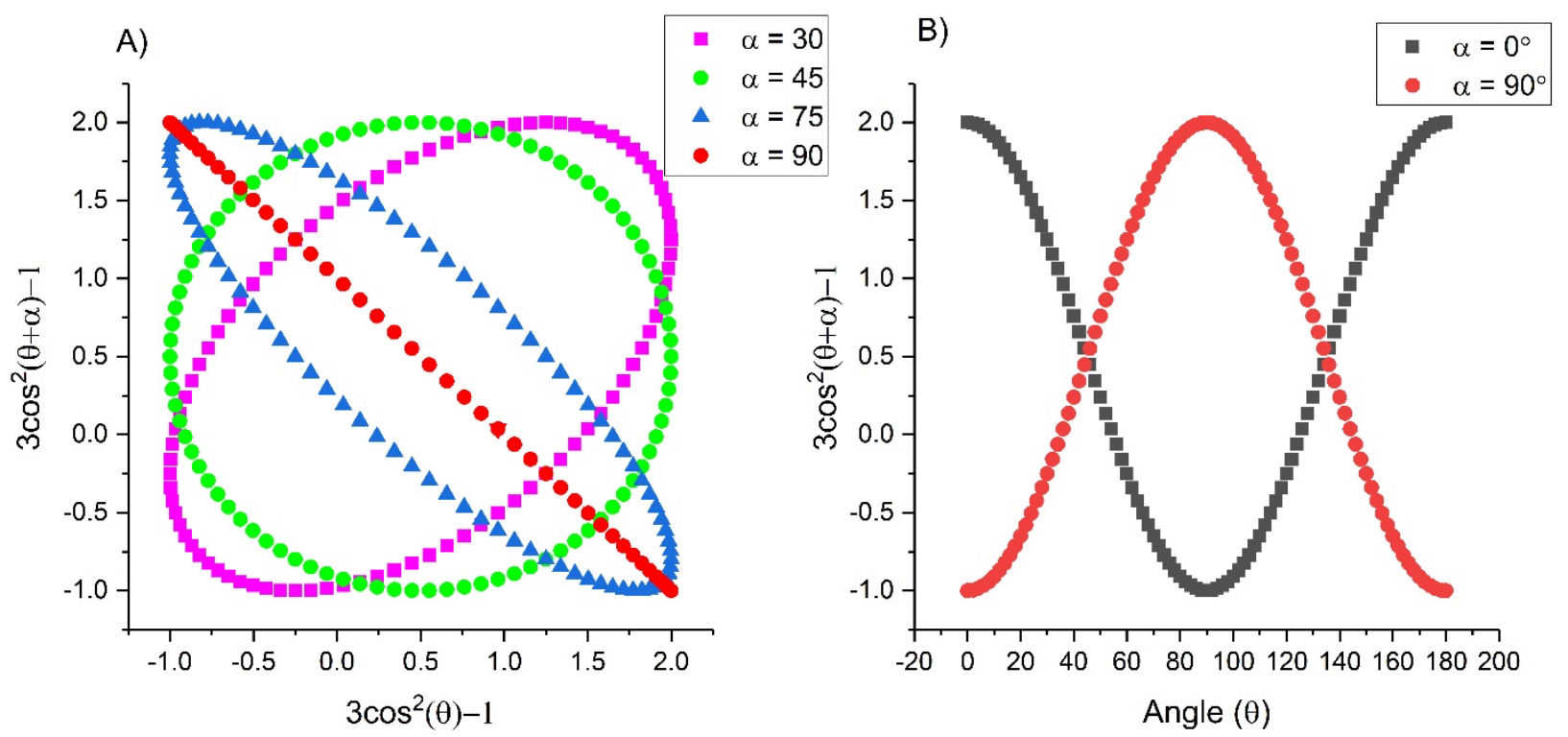

Figure S8. A) comparison of $3 \cos ^{2} \theta-1$ values as a function of the rotation angle $\alpha$. A linear correlation occurs for a rotation angle of $90^{\circ}$; where $\theta$ is the angle between the internuclear bond vector and the external magnetic field direction. B) Plot of $\left(3 \cos ^{2} \theta-1\right)$ as a function of $\theta$. 


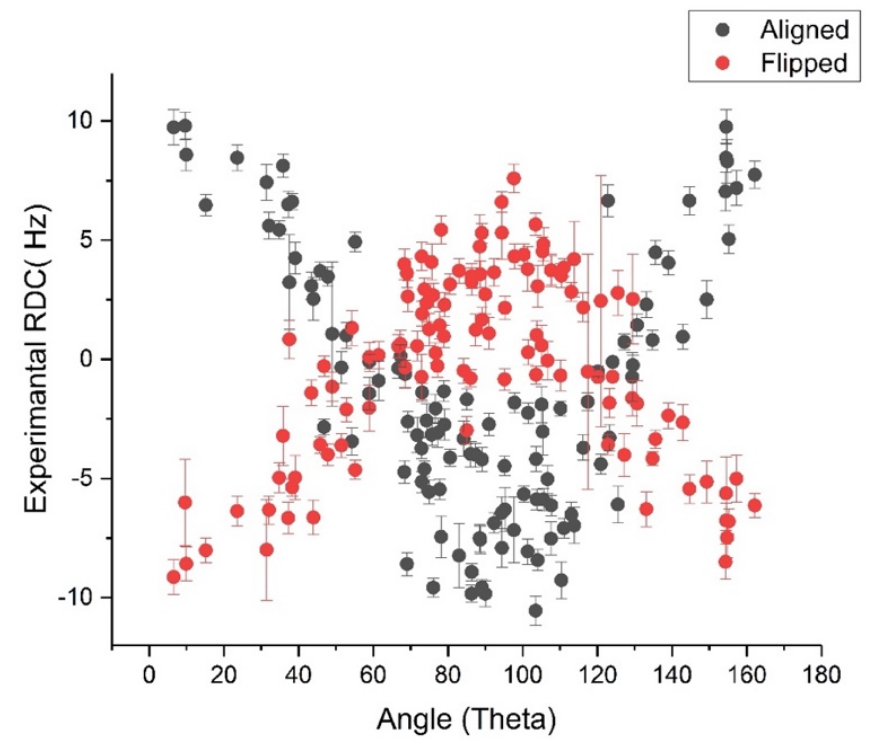

Figure S9. Plot showing the experimental RDCs as a function of the angle $(\theta)$ between the $\mathrm{N}-\mathrm{H}$ bond vector and the external magnetic field direction. $\theta$ value for each experimentally measured RDC was calculated by orienting the protein structure using the alignment tensor in the PALES software. The overall pattern is similar to the ideal case shown in Figure S6B.

A) DMPC-SMA-EA ( Aligned)

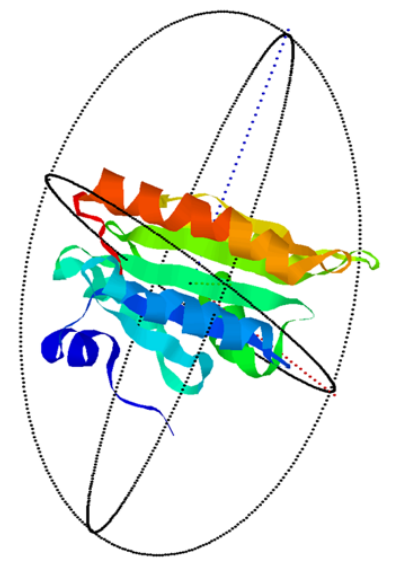

B) DMPC-DMPE-DTPA-Yb³+-SMA-EA ( Flipped)

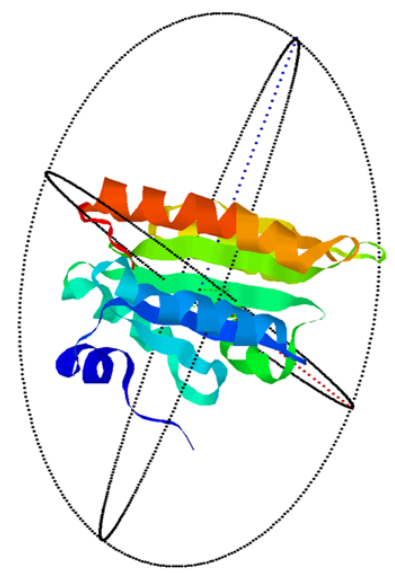

Figure S10. Graphical representation of alignment tensor measured from the singular value decomposition (SVD) method in the presence of aligned (A) and Flipped (B) nanodiscs as an alignment medium showing the alignment tensor is the same in the molecular frame of reference. The PALES software ${ }^{1}$ was used to generate the graphical depiction of alignment tensors. 
Table S1. Details of X-ray crystal structures of trFBD used for the comparison of the calculated and experimentally measured RDC values. The correlation plots are shown in Figures 4 and S7.

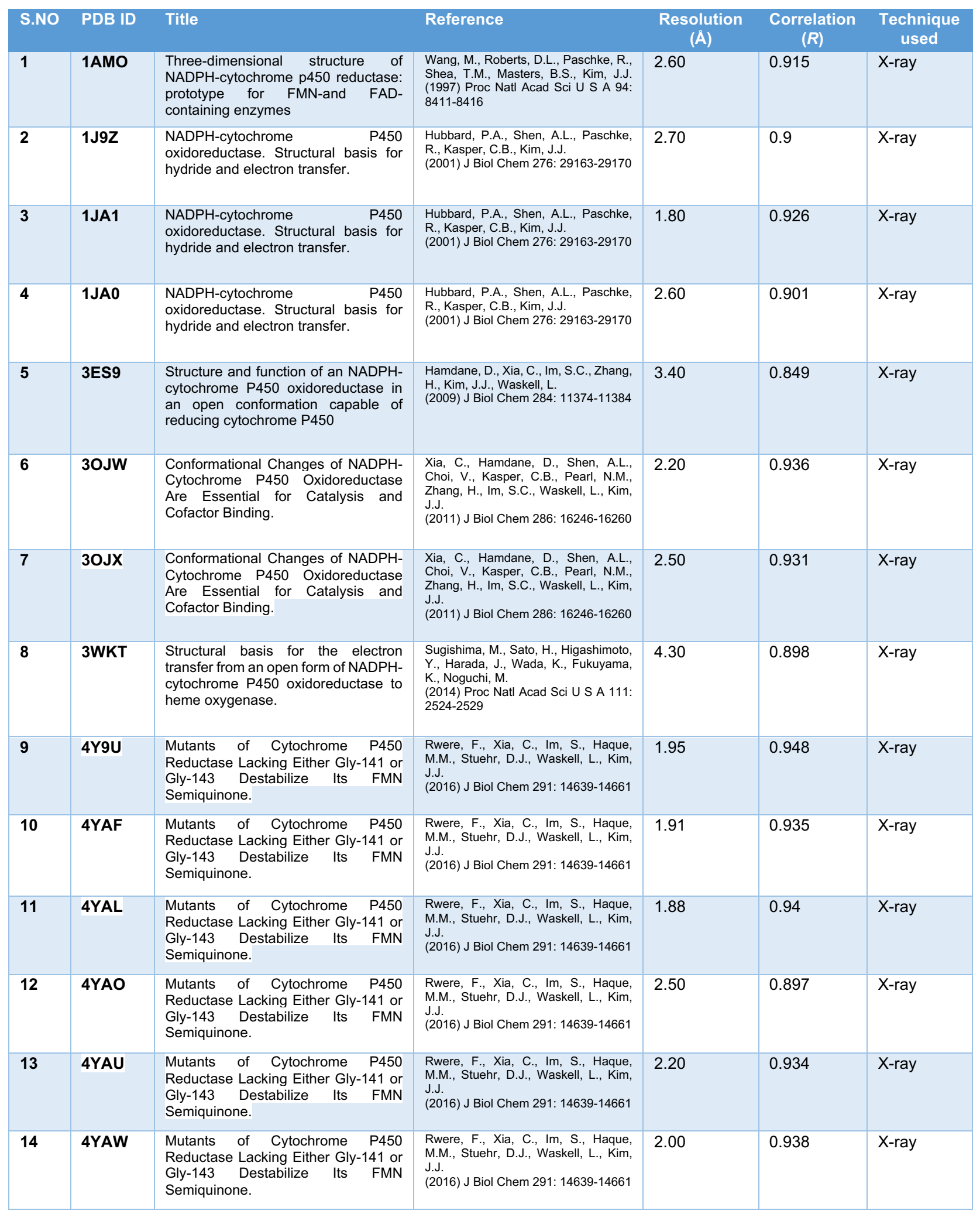




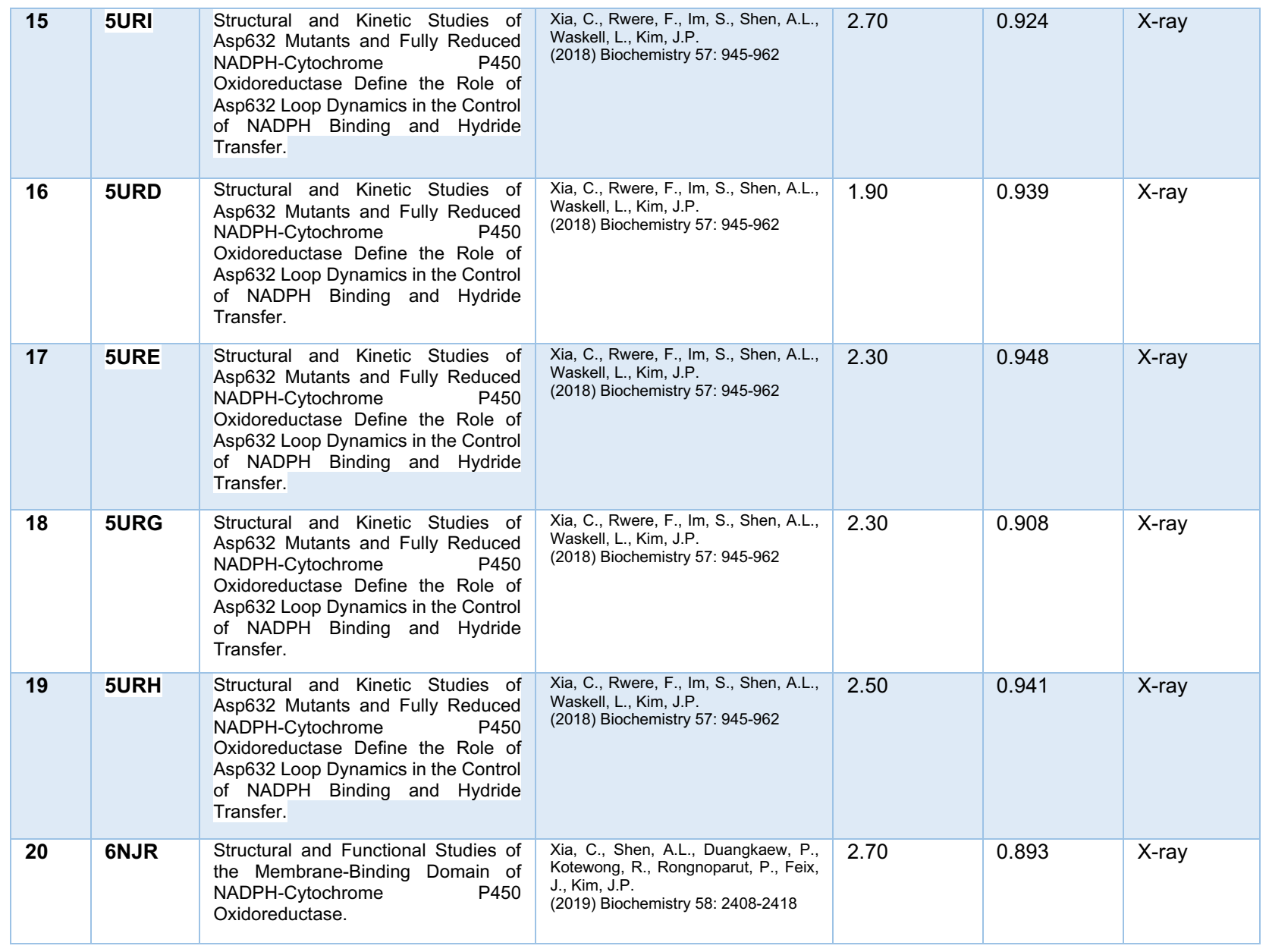


Table S2. The deviation of the difference in the RDC values for truncated-FBD. These values are The difference between the experimentally measured RDCS (using DMPC-SMA-EA nanodiscs) and the calculated RDCs using various crystal structures (in Table S1). These values are also plotted in Figure S5.

\begin{tabular}{|c|c|c|c|c|c|c|c|c|c|}
\hline & $1 \mathrm{AMO}$ & & $1 \mathrm{~J} 9 \mathrm{Z}$ & & $1 \mathrm{JAL}$ & & $1 \mathrm{JAO}$ & & $3 \mathrm{ES9}$ \\
\hline Glu66 & -5.5 & Glu66 & -6.9 & Glu66 & -6.9 & Glu66 & -7.1 & Met73 & -4.6 \\
\hline Met73 & -3.3 & Glu71 & -3.6 & Lys74 & 3.2 & Vla70 & -3.4 & Lys74 & 3.2 \\
\hline Phe83 & 3.2 & Lys74 & 3.2 & Phe83 & 3.2 & Glu71 & -4.4 & Thr76 & -3.5 \\
\hline Tyr84 & 4.2 & Thr76 & -4.2 & Ser86 & -5.8 & Met73 & -3.1 & Ile80 & -3.1 \\
\hline Ser86 & -4.2 & Phe83 & 5.0 & Arg104 & 5.2 & Lys74 & 4.0 & Ser86 & -3.1 \\
\hline Ser99 & -3.5 & Ser86 & -5.5 & Tyr105 & -6.2 & Thr76 & -7.1 & Gln87 & 3.8 \\
\hline Lys100 & -4.8 & Ser99 & -3.9 & Ala120 & -3.9 & Ser86 & -4.2 & Thr88 & 12.4 \\
\hline Tyr105 & -9.1 & Arg104 & 4.8 & Gly141 & -5.7 & Gln87 & 3.6 & Thr90 & -3.6 \\
\hline Gly106 & -6.2 & Met107 & -4.8 & Leu156 & 3.9 & Thr88 & 6.0 & Lys100 & -5.2 \\
\hline Met107 & -7.8 & Ala120 & -6.1 & Gly165 & -3.3 & Arg104 & 4.6 & Asp101 & -4.0 \\
\hline Asp118 & -3.8 & Ile128 & -3.1 & Gly172 & -3.3 & Tyr105 & -9.3 & Arg104 & 3.2 \\
\hline Ala120 & -5.7 & Ala138 & 3.8 & Asn175 & -3.8 & Ala138 & 5.5 & Gly106 & 5.7 \\
\hline Ala138 & 3.9 & Gly141 & -4.3 & Lys176 & -8.4 & Thr146 & -3.0 & Asp118 & -7.0 \\
\hline Glu158 & -3.1 & Asp154 & 3.5 & Gly196 & 5.3 & Glu158 & 5.2 & Ile128 & 3.1 \\
\hline leu163 & 5.5 & Gly172 & -3.3 & & & Gly165 & -3.5 & Thr146 & -6.0 \\
\hline Asn175 & -6.3 & Asn175 & -10.0 & & & Gly172 & -4.5 & Asp154 & 4.0 \\
\hline Thr177 & 4.2 & Tyr178 & -8.1 & & & Asn175 & -6.7 & leu163 & 7.4 \\
\hline \multirow[t]{14}{*}{ Gly196 } & 3.9 & Gly196 & 7.2 & & & Thr177 & 5.2 & Gly165 & -3.3 \\
\hline & & Ala197 & -3.1 & & & Gly196 & 5.5 & Val166 & 3.1 \\
\hline & & Gln198 & 4.3 & & & $G \ln 198$ & 4.2 & Ala169 & 4.7 \\
\hline & & & & & & & & Gly172 & -4.5 \\
\hline & & & & & & & & Gly174 & -3.2 \\
\hline & & & & & & & & Asn175 & -7.5 \\
\hline & & & & & & & & Thr177 & 4.8 \\
\hline & & & & & & & & Tyr178 & -4.4 \\
\hline & & & & & & & & Gly196 & 6.8 \\
\hline & & & & & & & & Ala197 & -4.6 \\
\hline & & & & & & & & Arg199 & 5.3 \\
\hline & & & & & & & & Leu205 & -4.3 \\
\hline & & & & & & & & Leu212 & -7.3 \\
\hline & & & & & & & & Gly232 & 3.4 \\
\hline
\end{tabular}




\begin{tabular}{|l|l|l|l|l|l|l|l|l|l|}
\hline & 3OJW & & 3OJX & & 3WKT & & 4Y9U & & 4YAF \\
\hline Glu71 & -3.2 & Glu71 & -3.9 & Glu71 & -3.0 & Glu66 & -5.7 & Glu66 & -5.2 \\
\hline Met73 & -4.0 & Lys72 & -3.3 & Met73 & -3.7 & Glu71 & -3.1 & Met73 & -3.2 \\
\hline Ile80 & -3.5 & Met73 & -3.6 & Thr76 & -3.1 & Lys74 & 3.7 & Lys74 & 3.9 \\
\hline Phe83 & 3.3 & Thr76 & -6.8 & Gly85 & -3.4 & Arg104 & 5.4 & Phe83 & 3.3 \\
\hline Ser86 & -4.9 & Ser86 & -4.7 & Ser86 & -4.5 & Ser111 & -3.0 & Ser86 & -3.7 \\
\hline Thr88 & 4.0 & Thr88 & 4.5 & Ser99 & -4.5 & Ala120 & -3.2 & Arg104 & 5.3 \\
\hline Arg104 & 5.5 & Ser99 & -3.7 & Tyr105 & -9.7 & Gly165 & -3.2 & Tyr105 & -7.6 \\
\hline Tyr105 & -3.5 & Arg104 & 4.7 & Gly106 & -6.8 & Gly172 & -3.0 & Ala120 & -4.2 \\
\hline Ala120 & -3.2 & Tyr105 & -4.3 & Met107 & -5.3 & Asn175 & -7.0 & Gly141 & -7.0 \\
\hline Gly141 & -7.7 & Ser131 & -3.1 & Asp118 & -5.0 & Gly196 & 5.0 & Leu156 & 3.1 \\
\hline Gly172 & -4.0 & Gly141 & -7.7 & Ala120 & -9.5 & & & Gly165 & -3.3 \\
\hline Asn175 & -5.5 & Thr159 & -3.0 & Leu125 & -4.7 & & & Gly172 & -3.2 \\
\hline Gly196 & 5.4 & Gly172 & -3.9 & Ile128 & -3.4 & & & Asn175 & -6.3 \\
\hline Gly232 & -4.6 & Leu173 & -3.9 & Thr146 & -4.5 & & & Gly196 & 5.5 \\
\hline & & Asn175 & -5.5 & leu163 & 4.3 & & & & \\
\hline & & Gly196 & 4.1 & Asn175 & -6.8 & & & & \\
\hline & & Gly232 & -4.7 & Lys176 & -3.5 & & & & \\
\hline & & & & Tyr178 & -3.4 & & & & \\
\hline
\end{tabular}

\begin{tabular}{|l|l|l|l|l|l|l|l|l|l|}
\hline & 4YAL & & 4YAO & & 4YAU & & 4YAW & & 5URI \\
\hline Glu66 & -4.4 & Glu66 & -6.6 & Glu66 & -4.9 & Glu66 & -4.7 & Glu66 & -6.9 \\
\hline Met73 & -3.2 & Glu71 & -3.2 & Glu71 & -3.3 & Glu71 & -3.1 & Glu71 & -3.5 \\
\hline Lys74 & 4.0 & Thr76 & -6.6 & Met73 & -3.5 & Met73 & -3.5 & Met73 & -3.4 \\
\hline Ile80 & -3.8 & Ile80 & -3.9 & Lys74 & 3.1 & Lys74 & 3.7 & Thr76 & 6.6 \\
\hline Ser86 & -4.8 & Ser86 & -4.3 & Thr76 & -3.7 & Ile80 & -3.5 & Tyr84 & 5.1 \\
\hline Thr88 & 3.1 & Thr88 & 6.2 & Ile80 & -4.6 & Ser86 & -4.4 & Ser86 & -4.5 \\
\hline Arg104 & 5.2 & Ser99 & -3.9 & Tyr84 & 3.4 & Thr88 & 5.9 & Arg104 & 5.5 \\
\hline Tyr105 & -6.8 & Arg104 & 4.9 & Ser86 & -4.4 & Arg104 & 4.9 & Tyr105 & -5.9 \\
\hline Ala120 & -3.4 & Ala120 & -4.6 & Arg104 & 5.6 & Tyr105 & -5.1 & Ala120 & -3.8 \\
\hline Gly141 & -5.5 & Ile128 & -6.7 & Tyr105 & -7.5 & Ala120 & -3.5 & Gly165 & -3.9 \\
\hline Gly165 & -3.1 & Asp129 & -3.2 & Ala120 & -3.3 & Gly143 & 4.5 & Leu173 & -3.1 \\
\hline Gly172 & -3.4 & Lys130 & 3.3 & Gly143 & 4.9 & Gly165 & -3.2 & Asn175 & -6.6 \\
\hline Asn175 & -6.1 & Asp154 & 3.6 & Gly172 & -4.1 & Gly172 & -4.1 & Tyr178 & -4.3 \\
\hline Gly196 & 5.3 & leu163 & 5.8 & Asn175 & -4.8 & Asn175 & -6.3 & Gly196 & 5.3 \\
\hline & & Val170 & 4.3 & Gly196 & 5.7 & Gly196 & 5.7 & & \\
\hline
\end{tabular}




\begin{tabular}{|l|l|l|l|l|l|l|l|l|l|}
\hline & Gly172 & -4.1 & & & & & & \\
\hline & & Gly174 & -3.0 & & & & & & \\
\hline & & Asn175 & -6.2 & & & & & & \\
\hline & Thr177 & 3.1 & & & & & & \\
\hline & Arg191 & -4.6 & & & & & & \\
\hline & Gly196 & 7.1 & & & & & & \\
\hline & & Leu212 & -3.7 & & & & & & \\
\hline & Phe223 & -3.2 & & & & & & \\
\hline & Val227 & -3.1 & & & & & & \\
\hline & Gly232 & -3.4 & & & & & & \\
\hline
\end{tabular}

\begin{tabular}{|c|c|c|c|c|c|c|c|c|c|}
\hline & 5URD & & 5URE & & 5URG & & $5 U R H$ & & 6NJR \\
\hline Glu66 & -4.4 & Glu66 & -6.0 & Glu66 & -4.9 & Glu66 & -5.2 & Glu66 & -4.6 \\
\hline Lys74 & 3.2 & Lys74 & 4.1 & Phe83 & 3.0 & Glu71 & -3.2 & Lys74 & 3.8 \\
\hline Ile80 & -3.3 & Ser86 & -4.7 & Ser86 & -5.5 & Met73 & -3.4 & Thr76 & -5.5 \\
\hline Ser86 & -4.4 & Thr88 & 3.1 & Thr88 & 3.2 & Lys74 & 3.9 & Ile80 & -3.5 \\
\hline Arg104 & 5.2 & Arg104 & 5.3 & Arg104 & 4.9 & Ile80 & -4.2 & Tyr84 & 3.4 \\
\hline Ala120 & -4.7 & Met107 & -4.0 & Tyr105 & -6.4 & Tyr84 & 4.5 & Gln87 & -3.2 \\
\hline Gly141 & -7.3 & Ala120 & -4.1 & Ile128 & -4.6 & Ser86 & -4.4 & Thr88 & 8.9 \\
\hline Gly172 & -3.1 & Gly141 & -3.8 & Gly141 & -5.9 & Arg104 & 5.2 & Arg104 & 4.9 \\
\hline Asn175 & -4.8 & Gly165 & -3.4 & Gly165 & -3.1 & Tyr105 & -5.4 & Tyr105 & -4.5 \\
\hline \multirow[t]{12}{*}{ Gly196 } & 5.3 & Gly172 & -3.4 & Gly172 & -3.3 & Met107 & -4.8 & Ala120 & -4.4 \\
\hline & & Asn175 & -5.6 & Asn175 & -10.0 & Thr159 & -3.1 & Ala138 & 6.4 \\
\hline & & Gly196 & 5.3 & Lys176 & -6.7 & Gly165 & -3.5 & Gly141 & -7.9 \\
\hline & & & & Tyr178 & -4.2 & Gly172 & -3.1 & Gln157 & -3.1 \\
\hline & & & & Gly196 & 5.0 & Asn175 & -5.5 & Glu158 & 8.8 \\
\hline & & & & & & Gly196 & 5.0 & Ala169 & -4.0 \\
\hline & & & & & & Val227 & -3.4 & Asn175 & -3.1 \\
\hline & & & & & & & & Lys176 & -5.4 \\
\hline & & & & & & & & Tyr178 & -6.4 \\
\hline & & & & & & & & Gly196 & 5.2 \\
\hline & & & & & & & & Gly206 & -3.2 \\
\hline & & & & & & & & Asp208 & -3.5 \\
\hline
\end{tabular}

\section{References:}

1. Zweckstetter, M., NMR: prediction of molecular alignment from structure using the PALES software. Nat Protoc 2008, 3 (4), 679-90. 